Suresh Mani

Gautam S. Grover

Stacy G. Bike

\section{Linear viscoelastic behavior of copper phthalocyanine dispersions used in printing inks}

Received: 15 June 1995

Accepted: 3 May 1996

\author{
Dr. S. Mani (四) \\ Flint Ink Corporation. \\ 4600 Arrowhead Drive \\ P.O. Box 8609 \\ Ann Arbor, Michigan 48 107, USA \\ Dr. G.S. Grover \\ Cabot Corporation \\ Aurora, Illinois, USA \\ Prof. S. G. Bike (四) \\ Department of Chemical Engineering \\ University of Michigan \\ Ann Arbor, Michigan 48109, USA
}

\begin{abstract}
In this paper we describe the linear viscoelastic properties of copper phthalocyanine (CuPCN) dispersions that are used in the manufacturing of offset lithographic printing inks. Transmission electron microscopy shows that the primary pigment particles are rod-like and have sizes in the range of 10 to $300 \mathrm{~nm}$. Steady shear measurements show that the dispersions are Newtonian at a pigment volume fraction of 0.073 and become increasingly shear thinning as the pigment volume fraction is increased. The strong shear-thinning nature of these dispersions can be attributed to the highly flocculated nature of the dispersions, which is due to interparticle attractions. The structural complexity of the dispersions also results in an unexpected linear viscoelastic response. While at low frequencies $(0.1$ and $1.0 \mathrm{~Hz})$ the ex-
\end{abstract}

tent of the linear region decreases with increasing pigment concentration, at a higher frequency $(10 \mathrm{~Hz})$ the extent of the linear region increases with increasing pigment concentration. This increase in the linear region with increasing pigment concentration suggests that at higher frequencies the dispersion is less brittle, and that the rheological behavior is dominated by intra-aggregate associations. In addition, frequency sweeps show that the dispersions behave like a viscoelastic liquid at low pigment concentrations. However, at higher pigment concentrations (yet significantly lower than the maximum packing fraction) the dispersions behave like a cross-linking polymer at its gel point.

Key words Copper phthalocyanine dispersions - flocculation gelation threshold

\section{Introduction}

The rheological properties of colloidal dispersions in lowmolecular-weight polymer solutions like paints and printing inks can strongly influence their conditions of manufacturing, handling, and transport as well as their end-use properties. The observed rheological behavior, which is related to the interparticle interactions in the dispersion, can include viscoelastic behavior as well as their thinning, shear thickening, and apparent yield stresses. It is consequently important to characterize the rheological behavior of these dispersions to gain information about the interparticle interactions giving rise to such behavior, and to intelligently design processes and products for the dispersions.

Studies of the rheological properties of colloidal dispersions in low-molecular-weight polymer solutions have traditionally concentrated solely on the steady-shear flow behavior (see, for example, reviews by Metzner, 1985; Kamal and Mutel, 1985; and Gupta, 1994). More recently, such rheological studies have included linear viscoelastic 
measurements, and the utility of these measurements in characterizing interparticle interactions in colloidal dispersions has been demonstrated (Buscall et al., 1982; Tadros and Hopkinson, 1990; Liang et al., 1993). For example, attractive interparticle forces have been shown to give rise to a viscoelastic solid-like plateau in the storage modulus $G^{\prime}$ at low frequencies, while repulsive interparticle forces give rise to viscoelastic liquid-like behavior as seen by a decrease in both $G^{\prime}$ and the loss modulus $G^{\prime \prime}$ with decreasing frequency (Onogi and Matsumoto, 1981). In addition, it has been shown that rheological measurements probing the interactions between colloidal particles stabilized by adsorbed polymer chains qualitatively agree with direct force measurements between mica surfaces bearing adsorbed polymer chains (Luckham et al., 1991; Costello et al., 1992 a; Costello et al., 1992 b; Tadros et al., 1993).

Linear viscoelastic measurements can also be used to gain information about the microstructure formed in flocculated colloidal dispersions (de Kruif, 1990; Chen and Russel, 1991). Flocculated colloidal dispersions can display viscoelastic solid-like behavior through a frequency-independent $G^{\prime}$ (van der Aerschot and Mewis, 1992; Sonntag and Russel, 1987). In such systems, de Silva et al. (1990) found that $G^{\prime}$ scaled as $\phi^{n}$, where $\phi$ is the particle volume fraction. The value of this exponent provides information about the mechanism of particle aggregation and the strength of the interparticle forces, with higher values of $n$ indicating stronger interparticle interactions (Buscall et al., 1988). Similarly, Grant and Russel (1993) have shown the elastic modulus to scale with $\left(\phi-\phi_{g}\right)^{S}$, where $\phi_{g}$ is the particle volume fraction at which the dispersion gels and $s$ characterizes the structure. Again, this relationship is valid for dispersions with $\phi>\phi_{g}$. Similar studies on dispersions of magnetic and nonmagnetic iron oxide particles in mineral oil demonstrated the presence of two regimes for the scaling of $G^{\prime}$ with $\phi$, with a threshold value of the volume fraction $\left(\phi_{c}\right)$ separating the two regimes (Kanai et al., 1992). The exponent $n$ was significantly larger for dispersions with concentrations $\phi>\phi_{c}$ as compared to dispersions with concentrations $\phi<\phi_{c}$. In addition, linear viscoelastic measurements can provide detailed information about the characteristic dimension of the microstructure. Shih et al. (1990) developed a scaling theory for colloidal gels that are well above the gelation threshold by modeling the gel network as a collection of flocs (fractal objects) and demonstrated that the fractal dimension of the flocs agreed well with that measured by static light scattering.

Most of these rheological studies probing interparticle interactions have concentrated on model systems consisting of spherical, monodisperse particles, a monodisperse stabilizing polymer, and a low-molecular-weight dispersing solvent. What is lacking is a systematic study of the linear viscoelastic behavior of more complex, industrially relevant dispersions that typically contain poly- disperse particles that are irreversible aggregated due to strong interparticle attractions and a dispersing polymer matrix that consists of one or more polymers and solvents.

In this paper, we examine the linear viscoelastic behavior of copper phthalocyanine (CuPCN) particles dispersed in a low-molecular-weight polymer solution. These dispersions of CuPCN particles, the most common blue pigment, are used in the manufacturing of offset lithographic inks. We use these data to characterize the interparticle interactions that affect the flow properties of this pigment dispersion.

\section{Materials and methods}

\section{Materials}

The dispersions studied were based on the copper phthalocyanine $(\mathrm{CuPCN})$ pigment. For more information about the synthesis, surface properties, and dispersion of the pigment, the reader is referred to Honigmann and Horn (1973), Fryer et al. (1981), McKay and Mather (1987), Smith (1988), and Clayton (1988). The characterization of the CuPCN pigment used in this study is described later in this section.

Dispersions of CuPCN at volume fractions of 0.073 , $0.154,0.239$, and 0.283 were obtained from the Pigments Laboratory, Flint Ink Corporation. These volume fractions were calculated by measuring the density of the pigment using ASTM D153 and density of the suspending medium using a weight-per-gallon cup. The suspending polymer matrix for these dispersions is a mixture of two polymeric liquids, an alkyd and a varnish whose principal component was a rosin-based resin, added in a volume ratio of $1: 3$, respectively. ${ }^{1}$ The molecular weight distributions of the two polymeric liquids were determined by gel permeation chromatography using polystyrene standards. The alkyd had a number-average molecular weight $M_{n}$ of 2405 and a weight-average molecular weight $M_{w}$ of 13286 , giving a polydispersity index of 5.5. The rosinbased resin was characterized by $M_{n}=2427$ and $M_{w}=2786$, giving a polydispersity index of 1.15 . The polymeric matrices were Newtonian for shear rates from 1 to $100 \mathrm{~s}^{-1}$, with an average viscosity of $18.4 \mathrm{~Pa} \cdot \mathrm{s}$. Linear viscoelastic measurements on the polymeric liquids showed that $G^{\prime}$ was an order of magnitude lower than $G^{\prime \prime}$, confirming the lack of any network structure in the absence of added pigment particles. Rothstein (1964)

\footnotetext{
1 For more information about alkyds and varnishes used in printing inks, the reader is referred to "The Printing Ink Manual," ed. F. A. Askew, 2nd edition, W. Heffer and Sons Ltd, 1969, pp. $622-664$
} 


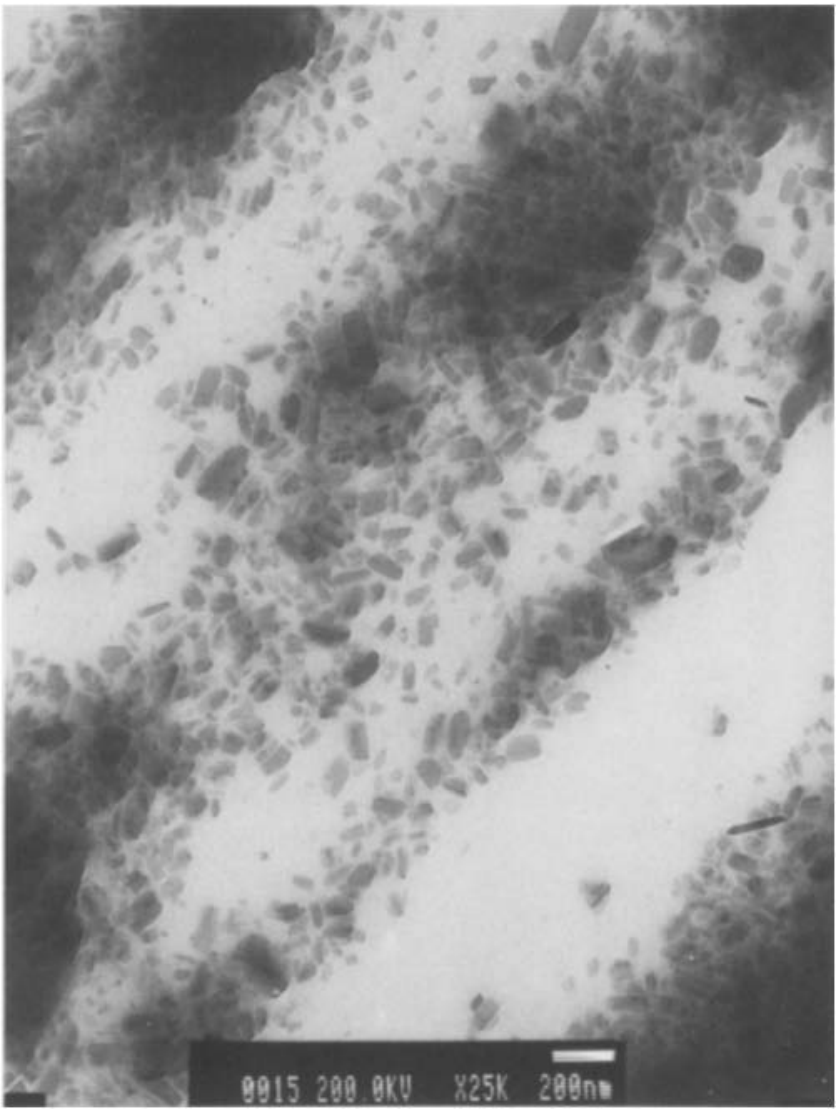

Fig. 1 Transmission electron micrograph of $\mathrm{CuPCN}$ particles at $25000 \times$

has demonstrated that alkyd resins similar to those used in this study adsorb onto CuPCN particles.

The CuPCN particles were characterized by transmission electron microscopy (TEM). Special sample preparation techniques were required to obtain sections that were transparent to electrons. Freshly cleaved mica was coated with carbon, onto which a thin layer of the dispersion was deposited using a razor blade. The mica sheet was then submerged in water, causing the carbon film coated with the dispersion to lift off of the mica. The sections were then captured on standard carbon grids and observed in a JEOL $2000 \mathrm{EX}$ transmission electron microscope. An electron micrograph of the particles is shown in Fig. 1. The primary particles, many of which are rod-like in shape, range from 10 to $300 \mathrm{~nm}$ in size, and some aggregation is noted. The large darker regions in the micrograph are most likely due to the polymer matrix. The matrix is also found near some of the particles making their boundaries less distinct.

The particle size was also measured using a Microtrac UPA-150 (Leeds and Northrup) which employs the principle of dynamic light scattering in the heterodyne configuration. The dispersions were diluted in toluene to yield solutions of approximately $1 \mathrm{wt} \%$ for analysis. As shown in Fig. 2, the measured sizes range from 0.1 to $4 \mu \mathrm{m}$, and more than $90 \%$ of the particles are less than $1 \mu \mathrm{m}$ in size. The larger particle size measured by this method reflects aggregation of the particles.

\section{Methods}

The rheological measurements were conducted on the Bohlin VOR (controlled strain rate) rheometer at $30 \pm 0.4^{\circ} \mathrm{C}$ using a cone-and-plate geometry (cone angle of $5^{\circ}$ and plate diameter of $30 \mathrm{~mm}$ ). Since the samples were sensitive to their deformation history, a suitable preshear followed by a relaxation period was required to obtain reproducible results. Preshearing the dispersions using large amplitude oscillatory shear (LAOS) at a frequency of $10 \mathrm{~Hz}$ and a strain amplitude of $0.207(100 \%$ on the Bohlin VOR) showed that $G^{\prime}$ initially decreased with time but then settled to a steady value after $100 \mathrm{~s}$. In contrast, preshearing at a constant shear rate led to alternate increases and decreases in viscosity and a suitable
Fig. 2 Particle size distribution of the CuCPN pigment measured using a Microtrac UPA-150

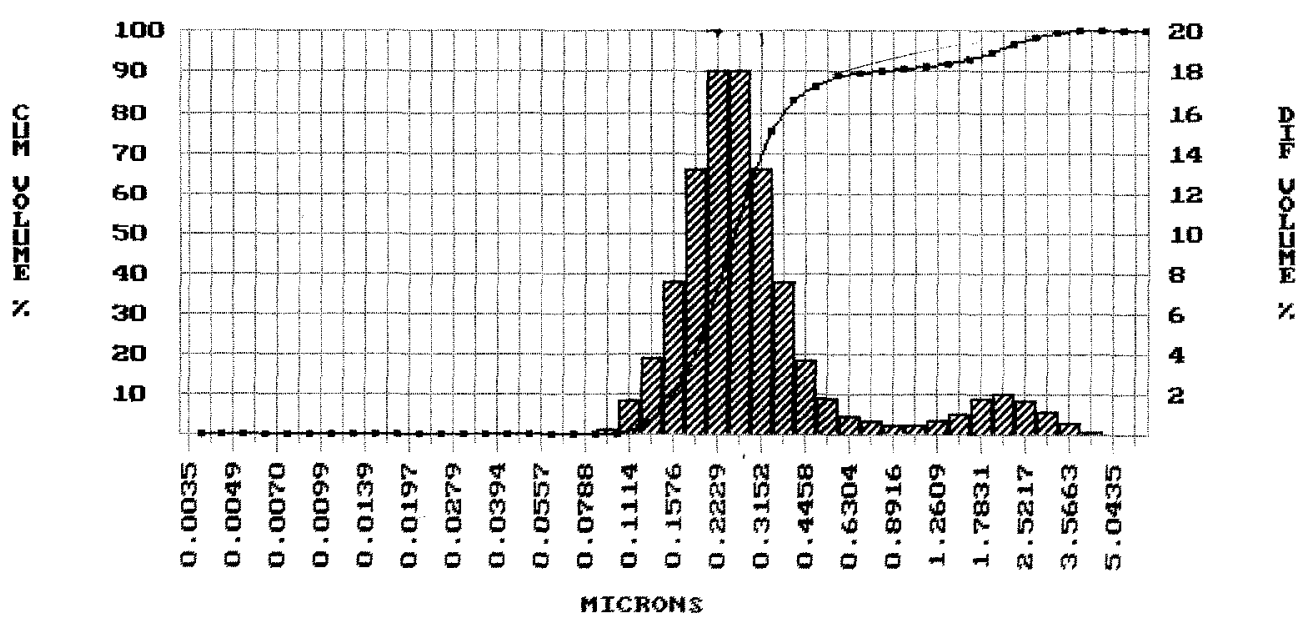


steady state was not reached during the $1000 \mathrm{~s}$ test period. This alternate increase and decrease in viscosity is indicative of anisotropic shear-induced structure within the dispersion (Hoffman, 1974; Gadala-Maria and Acrivos, 1980 ), and suggests the presence of strong interparticle forces between the CUPCN particles. Therefore, all samples were subjected to LAOS followed by relaxation for $1800 \mathrm{~s}$ prior to conducting any measurements; this time period was determined to be significantly longer than the longest relaxation time for any of the dispersions.

The viscosities of the dispersions were measured over a range of shear rates $\left(0.01\right.$ to $\left.50 \mathrm{~s}^{-1}\right)$; shear fracture and sample loss did not allow measurements at higher shear rates. In addition, the linear viscoelastic region was determined at each volume fraction by conducting strain sweeps at three different frequencies $(0.1,1.0$, and $10 \mathrm{~Hz})$, where the sample was subjected to increasing strains from $0.1 \%\left(2.07 \times 10^{-4}\right)$ to $100 \%\left(2.07 \times 10^{-1}\right)$. The sample was considered to be in the linear viscoelastic range for all strains until $G^{\prime}$ decreased to $5 \%$ below its initial value. Dynamic mechanical measurements were then conducted in the linear viscoelastic region at frequencies ranging from 0.01 to $10 \mathrm{~Hz}$. Measurements of the moduli were reproducible to within approximately $10 \%$ between repeat experiments, with the greatest deviations noted at lower frequencies; representative data are shown in Fig. 3.

Since slip of the dispersion at the plate interface is of concern, we also conducted small amplitude oscillatory shear measurements on these dispersions using a parallel plate geometry (Yoshimura and Prud'homme, 1988). The values of the moduli were statistically equivalent at plate

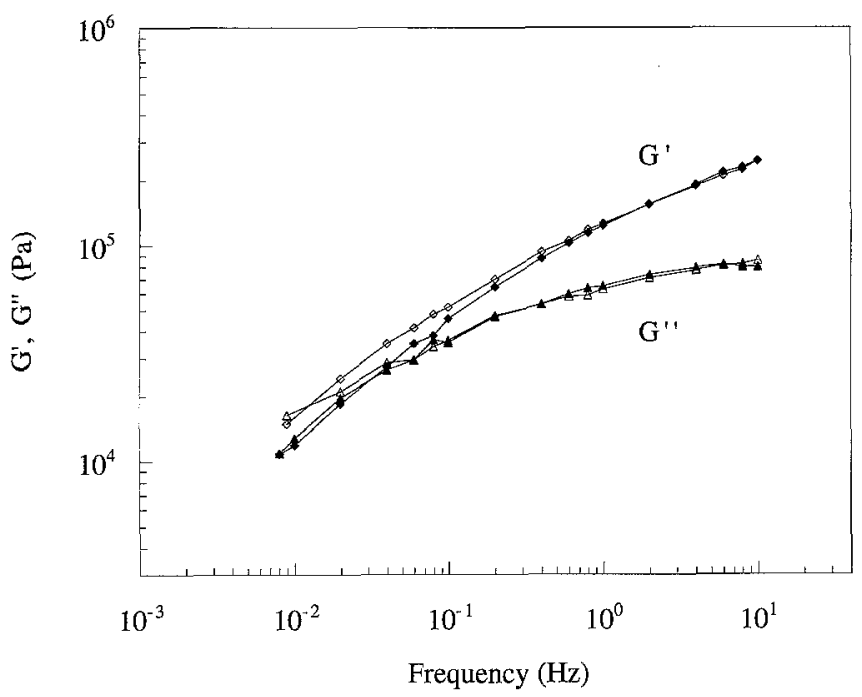

Fig. 3 Reproducibility of the rheological measurements, showing the storage modulus $G^{\prime}$ and the loss modulus $G^{\prime \prime}$ as a function of frequency $(\phi=0.283)$ for two consecutive runs. The closed symbols are data from the first run and the open symbols are data from the second run

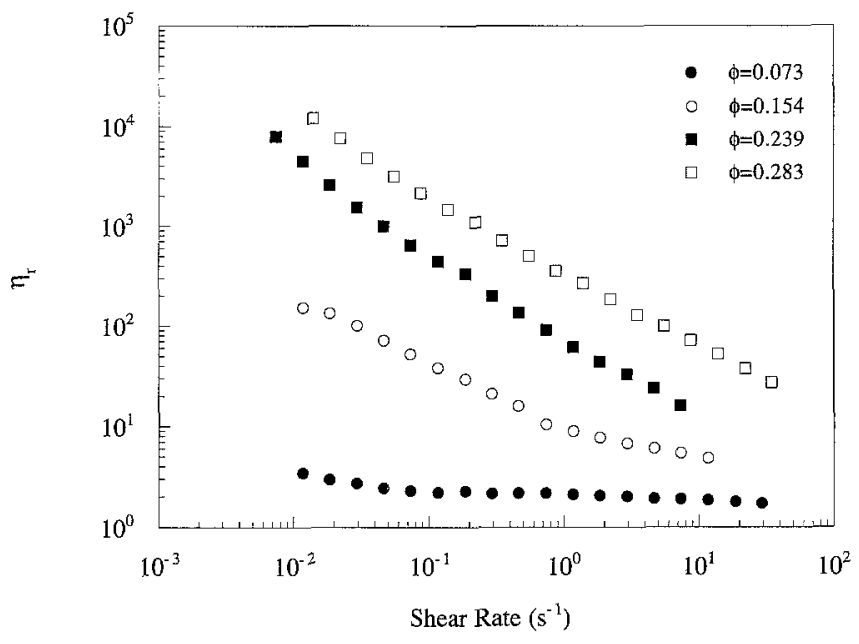

Fig. 4 Relative viscosity $\eta_{r}$ as a function of shear rate $\dot{\gamma}$ and pigment volume fraction $\phi$

separations of 0.5 and $1.5 \mathrm{~mm}$, demonstrating that slip was not significant with these dispersions at the particle concentrations studied.

\section{Results and discussion}

Shown in Fig. 4 are the relative viscosities of the dispersions as a function of shear rate, where the relative viscosity $\eta_{r}$ is the ratio of the viscosity of the dispersion $\eta$ to the viscosity of the dispersing (Newtonian) polymer matrix $\eta_{0}$. Plotting the relative viscosity against the shear rate eliminates the effect of the polymer matrix viscosity when comparing the steady shear behavior at different particle concentrations. The dispersions are Newtonian at a CuPCN volume fraction $\phi=0.073$ and are shear thinning at higher volume fractions in the shear rate range studied, with the degree of shear thinning increasing with particle concentration as expected.

Apparent yield values for the dispersions were estimated using the Casson equation (Casson, 1959), developed to describe the flow of printing inks:

$$
\tau^{1 / 2}=\tau_{c}^{1 / 2}+\eta_{c}^{1 / 2} j^{1 / 2},
$$

where $\tau$ is the stress, $\tau_{c}$ is the Casson yield value, $\eta_{c}$ is the Casson viscosity, and $j$ is the shear rate. Casson yield values calculated from the steady shear data are given in Table 1 . The Casson yield value scaled by the polymeric matrix viscosity exhibits a power-law dependence on particle volume fraction $\left(\tau_{c} / \eta_{0} \propto \phi^{n}\right)$, with a power-law index $n$ of 7.2. Power-law indices of 2.5 to 4.4 have been noted for weakly (reversibly) flocculated dispersions (van der Aerschot and Mewis, 1992; Boggs et al., 1996). The existence of an apparent yield value and the shear thinning noted at these relatively low volume fractions and 
Table 1 Casson yield values $\left(\tau_{C}\right)$ estimated from steady-shear viscometry

\begin{tabular}{lr}
\hline$\phi$ & $\tau_{C}(\mathrm{~Pa})$ \\
\hline 0.073 & 0.2 \\
0.154 & 37.8 \\
0.239 & 813.3 \\
0.283 & 3269.0 \\
\hline
\end{tabular}

the relatively high value of the power law index can be attributed to the highly flocculated nature of these dispersions (Macosko, 1994). Indeed, it is known that CuPCN particles experience strong interparticle attraction due to van der Waals forces, which leads to flocculation and a loss of color strength if not countered by a repulsive force (Moser and Thomas, 1963). This structure in the dispersions was studied further using dynamic oscillatory measurements.

The linear viscoelastic region of each dispersion was identified by conducting strain sweeps at different frequencies. Frith et al. (1987) have remarked that the linearity limit for dispersions is first noticed by a decrease in $G^{\prime \prime}$ rather than in $G^{\prime}$. For the CuPCN dispersions, however, both moduli decreased almost simultaneously as the strain was increased beyond the linear region. These strain sweeps (Fig. 5a-b) show that the extent of the linear viscoelastic region is strongly influenced by both particle concentration and frequency. Figure 5 a shows the values of $G^{\prime}$ for all dispersions as a function of strain at a frequency of $1 \mathrm{~Hz}$. At this frequency, the extent of the linear region decreases with increasing pigment concentration; the same trend was noted at a frequency of $0.1 \mathrm{~Hz}$. In contrast, the strain sweeps at $10 \mathrm{~Hz}$ (Fig. 5b) unexpectedly show an increase in the extent of the linear region with increasing pigment concentration.

Little theoretical and experimental work has been reported in the literature that supports the existence of an increase in the limit of linearity with increasing particle concentration. Shih et al. (1990) have proposed a scaling theory to describe the elastic properties of colloidal gels for particle concentrations well above the gelation threshold. While none of the dispersions described in this paper are "well above" the gelation threshold (and as will be noted later, only at the two higher volume fractions can the CuPCN dispersions be considered to be weakly gelled), some of the ideas from this scaling theory can be qualitatively applied to the results. Shih et al. proposes that when inter-aggregate bonds are stronger than intraaggregate bonds the limit of linearity should decrease with particle volume fraction; conversely, when intra-aggregate bonds are stronger than inter-aggregate bonds the limit of linearity should increase with particle volume fraction. This analysis suggests that intra-aggregate associations may govern the rheological behavior of the $\mathrm{CuPCN}$ dispersions at the frequency of $10 \mathrm{~Hz}$.

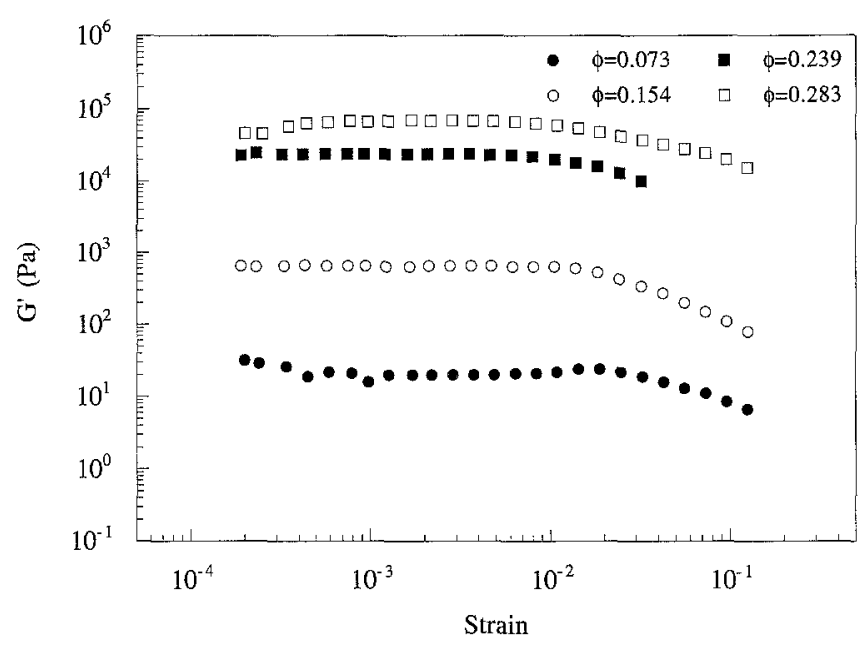

Fig. $5 \mathrm{~A}$ The storage modulus $G^{\prime}$ as a function of strain and pigment volume fraction $\phi$ at a frequency of $1.0 \mathrm{~Hz}$

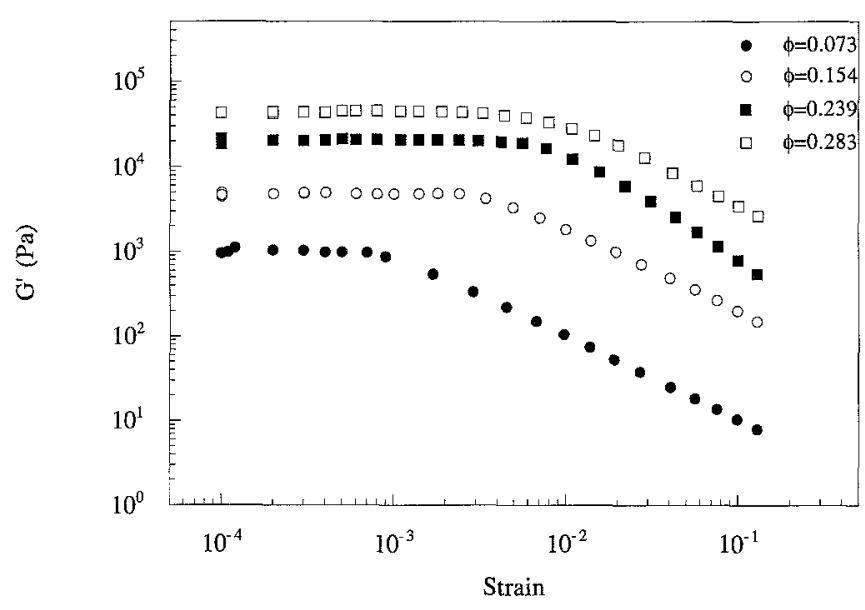

Fig. 5B The storage modulus $G^{\prime}$ as a function of strain and pigment volume fraction $\phi$ at a frequency of $10 \mathrm{~Hz}$

With regard to experimental data, Jones et al. (1991, 1992) noted a slight increase in the limit of linearity with increasing particle concentration above a particularly high value of $\phi$ ( 0.58 for Stöber silica stabilized by grafted octadecanol ("hard spheres"); 0.67 for copolymer particles stabilized by adsorbed poly(ethylene oxide) ("soft spheres")); below this value of $\phi$, the limit of linearity decreased with increasing particle concentration. The frequency dependence of the linearity limit was not discussed in that work, however. The experimental data showing an increase in the linear region with increasing concentration at the lower concentrations used in this study (below the gelation threshold) have not been previously noted in the literature, and provide an area for future study. 
In addition, Shih et al. suggests that the limit of linearity (strain) can be related to particle volume fraction by a power-law relationship for particle concentrations above the gelation threshold. While only the higher volume fraction dispersions are weakly gelled, we found a power-law dependence of the linearity limit on particle volume fraction. Based on the data given in Fig. $5 \mathrm{a}, \mathrm{b}$, the power-law index is -1.0 at a frequency of $1 \mathrm{~Hz}$ and +1.2 at a frequency of $10 \mathrm{~Hz}$. While these values of the powerlaw index are less than those seen by Shih (and this is to be expected, given that the dispersions are at best weakly gelled), the change in sign indicates a change in the mechanism of energy dissipation by the particle/polymer fluid networks. The data suggests that there are two different (frequency-dependent) mechanisms with two different length scales, and that at higher frequencies the length scale characteristic of intra-aggregate associations is dominant.

The nature of the linear viscoelastic response of these dispersions also depends on the pigment concentration. While the polymeric matrix had a Newtonian response with no inherent structure, adding pigment particles to the matrix creates a network structure within the dispersion leading to a progressively more elastic response at higher pigment concentrations. These network structures result from the interparticle forces (van der Waals and steric forces) that trap the polymeric fluid between the particles (Hunter, 1982).

At low pigment concentrations ( $\phi=0.073$ ), the dispersions exhibit a typical viscoelastic liquid-like response (Fig. 6a): $G^{\prime}$ and $G^{\prime \prime}$ have slopes close to 2 and 1 and $\tan \delta$ is on the order of 90 at low frequencies. At higher pigment concentrations (Fig. $6 \mathrm{~b}$ and c), $G^{\prime}$ and $G^{\prime \prime}$ display a power-law dependence on frequency over two decades of frequency and $\tan \delta$ is virtually independent of the frequency (note that we were not able to access the terminal region of these dispersions). At $\phi=0.154$ (Fig. 6b), $G^{\prime}$ and $G^{\prime \prime}$ are nearly parallel to each other over two decades of frequency and $\tan \delta$ is approximately constant at a value of 3. At $\phi=0.239$ (Fig. 6c), $G^{\prime}$ and $G^{\prime \prime}$ are nearly equal and the $\tan \delta$ is on the order of unity for frequencies less than $0.1 \mathrm{~Hz}$. At frequencies greater than $0.1 \mathrm{~Hz}, G^{\prime}$ is larger than $G^{\prime \prime}$ and $\tan \delta$ is less than unity. The small fluctuation in the data seen at approximately $0.06 \mathrm{~Hz}$ is probably due to a much larger-than-average aggregate, which is infrequently found in these commercially prepared concentrated dispersions; we do not believe that this fluctuation is indicative of a phase transition or any other similar phenomenon.

A similar frequency-independent $\tan \delta$ has been observed over five decades of frequency for cross-linking polymers at their critical extent of reaction or "gel point" (Chambon and Winter, 1986). Hence, the response of our dispersions appears to be intermediate to a viscoelastic liquid and a viscoelastic solid even at very low pigment volume fractions. Similar findings of gelation at low solid

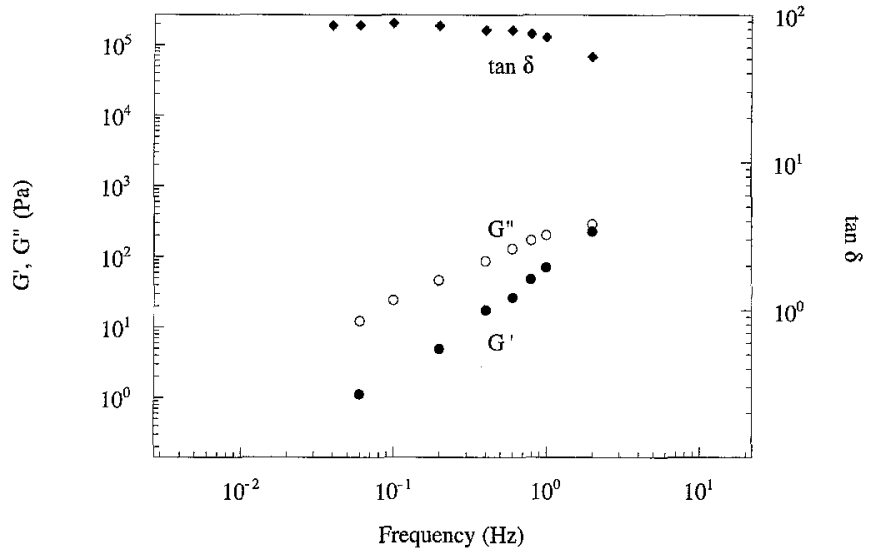

Fig. 6A The storage modulus $G^{\prime}$ and the loss modulus $G^{\prime \prime}$ as a function of frequency for a pigment volume fraction $\phi$ of 0.073

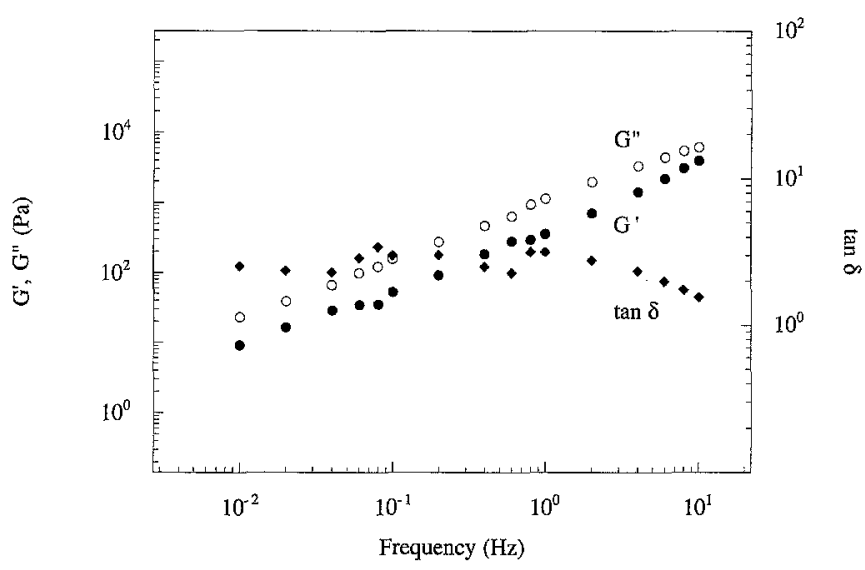

Fig. 6B The storage modulus $G^{\prime}$ and the loss modulus $G^{\prime \prime}$ as a function of frequency for a pigment volume fraction $\phi$ of 0.154

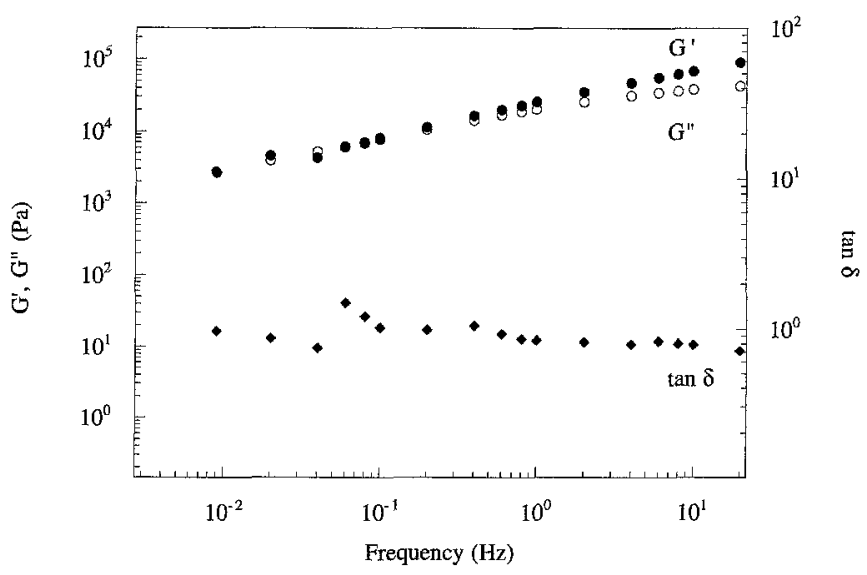

Fig. 6C The storage modulus $G^{\prime}$ and the loss modulus $G^{\prime \prime}$ as a function of frequency for a pigment volume fraction $\phi$ of 0.239 
volume fractions have been reported by Choi and Krieger (1986) using dispersions of sterically stabilized poly(methyl methacrylate) particles in siloxane fluids. Dispersions of larger particles in lower molecular weight siloxane fluids exhibited fluid-like rheological behavior, while dispersions of the particles in higher molecular weight siloxane fluids exhibited gel-like rheological behavior. The gel-like behavior was attributed to steric-elastic repulsion, resulting from the compression of the siloxane chains in the void spaces between the particles. In contrast, the transition from viscoelastic liquid-like to viscoelastic solid-like behavior for the soft spheres studied by Jones et al. (1992) occurred at much higher volume fractions than seen with the CuPCN dispersions, near the hard-sphere maximum packing fraction.

The gelation noted in the CuPCN dispersions at lower volume fractions likely results from strong interparticle attraction (due to van der Waals forces) relative to the steric repulsion due to adsorbed macromolecules and subsequent flocculation, not due to steric-elastic repulsion since the polymers in the dispersing media are of lower molecular weight than those for which Choi and Krieger noted gel-like behavior. We have attempted to quantify the thickness of the adsorbed polymeric layer on the CuPCN particles using a variation of a technique described by Reetz et al. (1995), who quantified surfactant adsorption on palladium clusters using a combination of high-resolution TEM and scanning tunneling microscopy (STM). Using atomic force microscopy (AFM) coupled with TEM, we were not able to determine the thickness of the adsorbed steric layer primarily due to nonuniformity of the CuPCN aggregate surfaces. Consequently, further work with specially synthesized model dispersions, in which polymer adsorption can be characterized, is needed to support the hypothesis that the gelation results from strong interparticle attraction.

In previous work on sterically stabilized silica dispersions, we examined the dependence of $G^{\prime}$ on $\phi$ (Grover and Bike, 1994; Grover, 1995). These strongly flocculated systems showed a frequency-independent $G^{\prime}$ and hence viscoelastic solid-like behavior. The elastic modulus exhibited a power-law dependence on the particle volume fraction, and as expected the more strongly flocculated dispersions gave higher values of the power-law index $n$. Such an analysis, as suggested by the work of de Silva et al. (1990) and Grant and Russel (1993), cannot be applied to the dispersions studied here, however, since none displayed either a frequency-independent $G^{\prime}$ or a limiting value of $G^{\prime}$ at high frequencies within the accessible frequencies. If such values of $G^{\prime}$ were available, we would expect a similar scaling of $G^{\prime}$ with volume fraction as seen with the Casson yield value.

This work has illustrated the utility of rheological measurements in probing the interparticle interactions in a technologically complex dispersion. Further work with these dispersions will involve developing model systems to mimic the observed behavior, so as to isolate those factors critical to determining the flow properties of the dispersions.

\section{Summary}

We have studied the linear viscoelastic response of industrially relevant copper phthalocyanine dispersions that are used in the manufacturing of offset lithographic printing inks. These dispersions present many inherent complexities: irreversibly aggregated CuPCN particles that are polydisperse and irregularly shaped, strong interparticle attractions and a dispersing varnish that is also polydisperse. Characterizing the linear viscoelastic behavior of these dispersions provides an understanding of the interparticle attractions in such systems.

The extent of the linear viscoelastic region for these dispersions was dependent on both the pigment concentration and the frequency. At 0.1 and $1 \mathrm{~Hz}$, the extent of the linear decreased with increasing pigment concentration, while at $10 \mathrm{~Hz}$ it increased with increasing pigment concentration. In addition, the dynamic behavior showed that as the pigment concentration was increased, the response of the dispersion varied from viscoelastic liquidlike (at $\phi=0.073$ ) to that of a crosslinking polymer near its critical extent of reaction.

Acknowledgments We thank Flint Ink Corporation and especially Dave Harrison for encouraging this work. We also thank Bob Holland, Pigments Lab, Flink Ink for preparing the pigment dispersions and Dan Lawrence of Flint Ink for the electron micrographs. In addition, we thank Prof. Paul Rasmussen of the University of Michigan for the gel permeation chromatography measurements. Useful discussions with Prof. Henning Winter are acknowledged. 


\section{References}

Buscall R, Goodwin JW, Hawkins MW, Ottewill RH (1982) Viscoelastic properties of concentrated lattices. Part 1 - Theoretical analysis. J Chem Soc, Faraday Trans I 78:2889-2899

Buscall R, Mills PA, Goodwin JW, Lawson DW (1988) Scaling behavior of the rheology of aggregate networks formed from colloidal particles. $\mathrm{J}$ Chem Soc, Faraday Trans I 84:4249-4260

Casson N (1959) A flow equation for pigment-oil suspensions of the printing ink type. Rheology of disperse systems. Mill CC (ed) Pergamon Press, New York, pp 84-104

Charnbon F, Winter HH (1986) Analysis of linear viscoelasticity of a crosslinking polymer at the gel point. J Rheol 30: $367-382$

Chen M, Russel WB (1991) Characteristics of flocculated silica dispersions. J Colloid Interface Sci 141:565-577

Choi GN, Krieger IM (1986) Rheological studies on sterically stabilized model dispersions of uniform colloidal spheres. J Colloid Interface Sci 113: $101-113$

Clayton M (1988) Raw materials. The printing ink manual, 4th edition, Leach RH et al (eds) Van Nostrand Reinhold (International) Co Ltd, England, pp 109-281

Costello BA de L, Luckham PF, Tadros ThF (1992a) Investigation of the interaction forces of polymer-coated surfaces using force balance, rheology, and osmotic pressure results. Langmuir 8:464-468

Costello BA de L, Luckham PF, Tadros ThF (1992b) Investigations of the properties of aqueous sterically stabilized dispersions. J Colloid Interface Sci 152: $237-246$

de Kruif CG (1990) The rheology of colloidal dispersions in relation to their microstructure. Hydrodynamics of dispersed media. Hulin JP et al (eds) Elsevier Science Publishers, B. V. (North Holland) pp 79-101

de Silva GPHL, Luckham PF, Tadros ThF (1990) The stability of silica in nonaqueous by poly-2-vinyl pyridine/polytert-butyl styrene block copolymers. II. Rheological studies. Colloids Surfaces 50:263-279

Frith WJ, Mewis J, Strivens TA (1987) Rheology of concentrated suspensions: experimental investigations. Powder Tech $51: 27-34$
Fryer JR, McKay RB, Mather RR, Sing KSW (1981) The technological importance of the crystallographic and surface properties of copper phthalocyanine pigments. J Chem Tech Biotechnol 31:371-387

Gadala-Maria F, Acrivos A (1980) Shear induced structures in a concentrated suspension of solid spheres. J Rheol 24:799-814

Grant MC, Russel WB (1993) Volume-fraction dependence of elastic moduli and transition temperatures for colloidal silica gels. Phys Rev A 47:2606-2614

Grover GS (1995) Rheological studies of flocculation in sterically stabilized silica dispersions. $\mathrm{Ph} \mathrm{D}$ Dissertation, The University of Michigan

Grover GS, Bike SG (1995) Monitoring flocculation in situ in sterically stabilized dispersions using rheological techniques. Langmuir 11:1807-1812

Gupta RK (1994) Particulate suspensions. Flow and rheology in polymer composites manufacturing. Advani SG (ed) Elsevier Science Publishers, New York, pp 9-51

Hoffman RL (1974) Discontinuous and dilatant viscosity behavior in concentrated suspensions. II. Theory and experimental tests. J Colloid Interface Sci 46:491 - 506

Hunter RJ (1982) The flow behavior of coagulated colloidal dispersions. Adv Colloid Interface Sci 17:197-211

Honigmann B, Horn D (1973) $\alpha: \beta$ transformation of copper phthalocyanine in organic suspensions. Particle growth in suspensions. Smith AL (ed) Academic Press, New York, pp 283-294

Jones DAR, Leary B, Boger DV (1991) The rheology of a concentrated colloidal suspension of hard spheres. J Colloid Interface Sci 147:479-495

Jones DAR, Leary B, Boger DV (1992) The rheology of a sterically stabilized suspension at high concentration. J Colloid Interface Sci 150:84-96

Kamal MR, Mutel A (1985) Rheological properties of suspensions in Newtonian and non-Newtonian fluids. J Polym Eng $5: 293-382$

Kanai H, Navarette RC, Macosko CW, Scriven LE (1992) Fragile networks and rheology of concentrated suspensions. Rheol Acta 31:333-344

Liang W, Tadro ThF, Luckham PF (1993) Investigations of depletion flocculation of concentrated sterically stabilized latex dispersions using viscoelastic measurements and microscopy. J Colloid Interface Sci 158:152-158
Luckham PF, Ansarifar MA, Costello BA de L, Tadros ThF (1991) The relationship between interparticle forces and the bulk rheology of suspensions. Powder Technol 65:371-379

Macosko CW (1994) Rheology: principles, measurements, and applications. VCH Publishers, New York

McKay RB, Mather RR (1987) The influence of aggregate structure and wetting on the dispersibility of $\beta$-copper phthalocyanine pigments in viscous printing inks. Colloids Surfaces 27:175-186

Metzner AB (1985) Rheology of suspensions in polymeric liquids. $J$ Rheol 29:739-775

Moser FH, Thomas AL (1963) Phthalocyanine compounds. Reinhold Publishing Corporation, New York

Onogi S, Matsumoto T (1981) Rheological properties of polymer solutions and melts containing suspended particles. Polym Eng Rev 1:45-87

Reetz MT, Helbig W, Quaiser SA, Stimming U, Breuer N, Vogel R (1995) Visualization of surfactants on nanostructured palladium clusters by a combination of STM and high-resolution TEM. Science 267:367-369

Rothstein EC (1964) Role of the adsorbed resin layer in the dispersion of pigmented alkyd systems. Offic Dig Fed Soc Paint Technol 36:479-484

Sonntag RC, Russel WB (1987) Elastic properties of flocculated silica networks. J Colloid Interface Sci 116: $485-489$

Shih WH, Shih WY, Kim S, Liu J, Aksay IA (1990) Scaling behavior of the elastic properties of colloidal gels. Phys Rev A 42:4772-4779

Smith HM (1988) Phthalocyanine blue pigments. Pigment Handbook. Lewis PA (ed) John Wiley and Sons, New York, pp $663-674$

Tadros ThF, Hopkinson A (1990) Use of viscoelastic measurements for investigating the stability/flocculation of concentrated dispersions. Faraday Disc Chem Soc 90:41-55

Tadros ThF, Liang W, Costello B, Luckham PF (1993) Correlation of the rheology of concentrated dispersions with interparticle interactions. Colloids Surf A 79:105-114

van der Aerschot E, Mewis J (1992) Equilibrium properties of reversibly flocculated dispersions. Colloids Surfaces 69:15-22

Yoshimura A, Prud'homme RK (1988) Wall slip corrections for couette and parallel disk viscometers. J Rheol 32:53-67 